\title{
The effects of septal lesions on the learning and reversal of a kinesthetic discrimination'
}

\author{
PAUL L. GITTELSON ${ }^{2}$ and PETER J. DONOVICK, ${ }^{3}$ State \\ University of New York at Binghamton, N. Y. 13901
}

The effects of septal lesions on the learning and reversal behavior of the rat in a kinesthetically cued discrimination problem were examined. Septal lesions significantly impaired the reversal but not the original learning of the discrimination, supporting the notion that the septal region is involved in the inhibition of previously learned somatomotor cued responses.

The septal region of the forebrain apparently plays an essential role in the inhibition of previously established somatomotor cued responses (e.g., McCleary, 1961). Septal disfunction, produced either by lesions (e.g., Schwartzbaum \& Donovick, 1968) or low level electrical stimulation (e.g., Donovick \& Schwartzbaum, 1966) results in a great impairment of a spatial (position) discrimination and in the learning of a spatial alternation problem. The deficiency in response inhibition following septal lesions is also exhibited in passive-avoidance behavior (McCleary, 1961), the formation of a conditioned emotional response (Harvey, Lints, Jacobson, \& Hunt, 1965), and responding on fixed-interval schedules of reinforcement (Ellen \& Powell, 1962). It may be noted that the facilitation observed on an active avoidance problem (e.g., McCleary, 1961) may be interpreted as a deficit in response inhibition.

Apparently, however, there is some degree of specificity in the perseverative disorder which characterizes much of the septal lesioned Ss' behavior. That is, although septal lesions dramatically impair the inhibition of somatomotor cued behavior, they generally do not affect the reversal of a brightness discrimination (Schwartzbaum \& Donovick, 1968; but see Thompson, 1966). The present study is an attempt to further specify the nature of the perseverative disorder, by examining the learning-and reversal-of a somatomotor (kinesthetic) cued task which is independent of position. To this end, we studied the behavior of septal lesioned and sham operated rats on the acquisition and reversal of a kinesthetic discrimination in a modified T-maze $(15 \mathrm{deg}$ incline vs "horizontal" alleys) in which position of the inclined alley was counterbalanced between the two sides.

\section{METHOD}

The Ss were 15 male albino rats obtained from the Rolfsmeyer Co., weighing approximately $300 \mathrm{~g}$. The experimental Ss $(N=8)$ received bilateral electrolytic lesions of the septal region, while control $\mathrm{Ss}(\mathrm{N}=7)$ received sham operations. Surgery was performed under nembutal anesthesia using standard stereotaxic procedure, and lesion placement was verified at the end of testing. (See Donovick, Burright, \& Gittelson, 1968, for detail.)

Discrimination tests and pretraining were conducted in a modified T-maze. The arms of the maze were hinged so that they could be raised to a $15 \mathrm{deg}$ slope or left in the horizontal plane. The center alley of the maze was $30 \mathrm{in}$. long and each of the arms were 24 in. A start door was positioned $10 \mathrm{in}$. from the beginning of the center alley and goal box doors were placed $9 \mathrm{in}$. from the end of each arm. The alleys were $4 \mathrm{in}$. wide and the sides were $8 \mathrm{in}$. high. The entire maze was painted flat black and the maze was located in a quiet room illuminated only by a $25 \mathrm{~W}$ shielded red light bulb, thus minimizing if not eliminating the visual cues available to the rat.
Following surgery, Ss were placed on a limited food regime which maintained their bodyweight between $290-310 \mathrm{~g}$. One week after surgery, Ss were given eight pretraining trials per day for three days. Pretraining trials were balanced and "forced" trials were employed to insure that each $S$ received equal experience in each arm of the maze and with both arm positions. During pretraining, every response was rewarded with two 45-mg (Noyes $\mathrm{Co}$.) food pellets. Following the pretraining, Ss were randomly assigned to either the incline$(N=4$ septal and three control Ss) or horizontal- $(N=4$ septal and four control Ss) rewarded condition (two-pellet reward). The position of the elevated arm was counterbalanced between the right and left sides. The Ss were run for 24 trials per day until a criterion of 22 correct out of 24 responses was met. Reward conditions were then reversed and Ss run to criterion.

\section{RESULTS}

Histological verification of the placement of the lesions typically showed near total destruction of the septum from the region of the hippocampus pars anterior to a point just posterior to the crossing of the anterior commissure. Some lesions invaded the nucleus accumbens and anterior commissure but damage to other adjacent structures was essentially nonexistent.

In confirmation of previous investigations (e.g. Schwartzbaum \& Donovick, 1968), septal lesions impaired the reversal of a somatomotor cued problem without markedly affecting original learning of the task. When the rewarded response was reversed, control Ss returned to a chance level of performance (mean trials $=31.57$ ) sooner than lesioned (mean $=53.25)$ Ss $\left(U=13, n_{1} n_{2}=8,7 ; p=.047\right)$. Further, as shown in Table 1 , which presents the mean number of trials to criterion, septal lesioned $S s$, re control Ss, exhibited a considerable deficit in attaining criterion of the reversed kinesthetic discrimination $\left(U=2, n_{1} n_{2}=8,7 ; p=.001\right)$. That is, lesioned Ss made considerably more errors before mastering the problem. As a further indication of the perseverative tendencies of the septal Ss on reversal, a difference score was obtained for each $S$ by subtracting the number of trials to criterion on original learning from the number of trials to criterion on reversal. Again as can be seen from Table 1, the difference in the number of trials to criterion for original learning and reversal was considerably larger for the septal lesioned group $\left(U=14, n_{1} n_{2}=8,7 ; p=.06\right)$. (For both original learning and reversal, the septal lesioned and control Ss learned to approach the horizontal alley more rapidly than the inclined one.)

As in earlier studies (e.g., Schwartzbaum \& Donovick, 1968) we found no significant $\left(U=19, n_{1} n_{2}=8,7 ; p=.168\right)$

Table 1

Mean Number Responses to Criterion and Difference Scores

\begin{tabular}{llrrr}
\hline & & Mean & Range & SD \\
\hline S & O.L. & 94.88 & $75-121$ & 18.26 \\
E & Rev. & 145.38 & $116-169$ & 15.98 \\
P & Rev.0.L. & 50.50 & $16-79$ & 23.92 \\
T & & & $24-120$ & 41.44 \\
\hline C & O.L. & 74.00 & $77-125$ & 17.00 \\
O & Rev. & 101.43 & $-57-101$ & 56.06 \\
N & Rev. O.L. & 10.29 & & \\
T & & & & \\
R & & & & \\
\hline
\end{tabular}


impairment in the original learning of the task. The apparent difference shown on original learning (Table 1) may be attributed to the strong horizontal preference of two control Ss who learned the problem almost immediately (24 and 27 trials). Thus the present findings support the contention that the septal lesions produce a specific perseverative disorder rather than a general decrement in the learning ability.

$$
\text { DISCUSSION }
$$

The present investigation extended earlier findings (e g., Schwartzbaum \& Donovick, 1968) that septal lesions impair the reversal of a somatomotor cued response (e.g., spatial discrimination) without disturbing the original learning of the problem. Specifically, we found that septal lesions hinder the reversal of a kinesthetic discrimination in which the correct response was independent of position. Thus, this study in conjunction with others indicates that once the response is learned, the septal lesioned $S$ persisted in his execution of it, despite shifts in the rewarded response (reversal), the absence of reward (extinction) or, even when the response is punished (passive avoidance). It may be noted that the increased fluid consumption seen following septal lesions (e.g., Donovick, Burright, \& Gittelson, 1968) may be looked at as perseveration of a well-established behavior. It thus appears that septal lesioned Ss show no generalized decrement in their ability to learn a problem and only suffer a decrement in inhibiting certain well-established response tendencies even when that response may no longer be adaptive to the well-being of the $\mathrm{S}$. The present study demonstrates that the maladaptive behavior of septal lesioned rats can be seen when the learning is based upon kinesthetic cues even when this response is unrelated to the spatial cues of the goal.

The lesions employed in this study invaded the entire precommissural septal area and thus did not allow for anatomical fractionation of the region. However, research (Donovick, in press) suggests that the present perseverative effects are due primarily to disruption of the anteromedial septal nuclei, including the dorsal part of the diagonal-band system. Lesions confined to this area completely blocked hippocampal theta activity and produced a marked effect on the discrimination reversal (Donovick, in press). This suggests a common anatomical basis for changes in perseverative behavior following septal or hippocampal lesions.

\section{REFERENCES}

DONOVICK, P. J. Effects of localized septal lesions on hippocampal EEG activity and behavior in rats. Journal of Comparative \& Physiological Psychology, in press.

DONOVICK, P. J., BURRIGHT, R. G., \& GITTELSON, P. L. The effect of septal lesions on saccharine choice as a function of water deprivation. Physiology \& Behavior, in press.

DONOVICK, P. J., \& SCHWARTZBAUM, J. S. Effects of low-level stimulation of the septal area on two types of discrimination reversal in the rat. Psychonomic Science, 1966, 6, 3-4.

ELLEN, P., \& POWELL, E. W. Effects of septal lesions on behavior generated by positive reinforcement. Experimental Neurology, 1962, 6, 1-11.

HARVEY, J. A., LINTS, J., \& HUNT, H. F. Effects of lesions in the septal area on conditioned fear and discriminated instrumental punishment in the albino rat. Journal of Comparative \& Phy siological Psychology, 1965, 59, 49-56.

McCLEARY, R. A. Response specificity in the behavioral effects of limbic system lesions in the cat. Journal of Comparative \& Physiological Psychology, 1961, 54, 605-613.

SCHWARTZBAUM, J. S., \& DONOVICK, P. J. Discrimination reversal and spatial alternation associated with septal and caudate disfunction in rats. Journal of Comparative \& Physiological Psychology, 1968, 58 , 83-92.

THOMPSON, J. B. Reversal behavior in rats following lesions in the hippocampus. Unpublished dissertation, University of Wisconsin, 1966.

\section{NOTES}

1. We thank Professor R. G. Burright for reading this manuscript. This investigation was supported in part by NIMH Grant No. MH 14232-01 to the second author.

2. Present address: Brain Research Center, Syracuse University.

3. Reprint requests should be addressed to second author. 